\title{
DEVICE DESIGNED FOR DETECTION AND SETTING THE REQUIRED TENSILE FORCE IN ROPES
}

\author{
Leopold Hrabovský' ${ }^{\text {, Marinko Maslarić }}{ }^{2}$ \\ 1 VŠB - Technical University of Ostrava, 17. listopadu 15/2172, 70833 Ostrava - Poruba, Czech Republic, \\ e-mail: leopold.hrabovsky@vsb.cz \\ 2 Faculty of Technical Sciences, University of Novi Sad, Dr Zorana Đinđića 1, 21101 Novi Sad, Republic of Serbia, \\ e-mail: marinko@uns.ac.rs
}

Received: 2018.01.15

Accepted: 2018.02.01

Published: 2018.03.01

\begin{abstract}
The contribution deals with a device, which allows recording values of tensile forces acting on steel ropes. At the same time, this device allows setting of the same values of tensile forces in carrier ropes within multi-rope systems, which are initially unevenly subjected to tension by the action of the weight suspended on these ropes. In the contribution, two principles of devices for setting the required value of tensile force in ropes are mentioned and subjected to the analysis of practical applicability. The paper describes in detail the design and principle of operation of the mechanical device for setting the required value of tensile force in ropes, which uses screw drive and tensile force sensor.
\end{abstract}

Keywords: rope sensor, elevator rope, tension equalizer, lift

\section{APPLICABLE REGULATIONS AND REQUIREMENTS}

In 2014, the European Committee for Standardization issued two new safety regulations [2] and [3] for the construction of elevators and the inspection of their components. These regulations [2] and [3] have been in force since 2014, but only turned mandatory on 1 September, 2017, until that date they applied in parallel with the regulations [1].

Regulation [2] sets down technical requirements and safety rules for construction and installation of elevators for transport of persons and persons and freight. Regulation [3] defines constructing principles, calculations, inspections and tests of elevator components.

Regulation [1], which expired on 1.7.2010 and was replaced with [2] and [3], states in Chapter 9 that the elevator cages and balancing or offsetting weights must be suspended on steel ropes or steel chains with parallel links or on roller chains.
If steel ropes are used as elevator carrier components, then they must meet given requirements $[7,8,9]$. First of them is that the minimum nominal diameter of the carrier steel rope is at least 8 $\mathrm{mm}$. The second is the requirement for minimum value of the nominal tensile strength of the wire (especially for ropes with wires of the same tensile strength and ropes with two nominal tensile strengths of wires) $[10,11]$.

At least two cross-sections of the carrier ropes for suspension of the elevator cages must be used and the ratio of friction discs pitch diameters, pulleys and drums to the nominal carrier ropes diameter must be at least 40 , regardless the number of rope strands. [12, 13, 14]

\section{LEGISLATIVE REGULATIONS}

The above requirement according to [1] for the required ratio of friction disc pitch parameter to the nominal diameter of the carrier ropes re- 
quires the contractor of the elevators, due to the effort to reduce the space taken by the elevator, under approved conditions by the competent authorities, to use carrier ropes of smaller nominal diameters than $8 \mathrm{~mm}$.

The use of carrier ropes with smaller nominal diameters inevitably brings the requirement for the use of a higher number of ropes for the given loading capacity of the elevator. However, an increasing number of the cross-sections of carrier ropes lead to a possible situation, when the loading exerted by the weight of the cage and the weight and the weight of the counterweight is not evenly distributed into the individual cross-sections of the carrier ropes. This different loading of the individual carrier ropes leads to an uneven wear of the friction disc grooves and different wear of the individual carrier ropes. As for this condition [3] considers and requires the elevator contractor to test that the load is evenly distributed into all cross-sections of the carrier ropes.

\section{EXISTING PRINCIPLES OF DEVICES CAPABLE OF DETECTING TENSILE FORCES IN ROPES}

Several principles are currently known on the consumer market allowing subtraction of the acting tensile force in the ropes and eventually offsetting the different tensile force values in the individual ropes.

Well known is the principle of a rope sensor, see Figure 4, e.g. [4], measuring the tensile force in the rope using the principle of bending deformation of the beam loaded with single force, see Figure 1. This device uses bodies with a measuring member and three contact points in a plane. The measured rope runs between these points. The two outside contact points on the device body, located at a known distance apart, serve as support of the measured rope. The third contact point is located at the midpoint distance of the pitch of the two outside contact points, which is distanced by the $h$ value $[\mathrm{m}]$ from the axes intersection of the outside contact points, se Figure 2. Due to the acting tensile force $\mathrm{T}[\mathrm{N}]$ in the rope, a compressive force $\mathrm{F}[\mathrm{N}]$ is then exerted on the third contact point, see the relation (1), which is recorded by the measuring sensor.

$$
\mathrm{F}=2 . \mathrm{T} \cdot \sin (\alpha)[\mathrm{N}]
$$

According to Figure 2, the following dependencies can be determined between the relevant parameters:

$$
\begin{aligned}
& \alpha=\operatorname{arctg}(d / b)=\operatorname{arctg} \\
& {[(h+2 . R \cdot \cos \alpha) /(a-2 . R \cdot \sin \alpha)]} \\
& d=h+2 \cdot c=h+2 \cdot R \cdot \cos \alpha[m] \\
& c=R \cdot \cos \alpha[m] \\
& b=a-2 \cdot e=a-2 . R \cdot \sin \alpha[m] \\
& e=R \cdot \sin \alpha[m]
\end{aligned}
$$

Obtaining the value of the tensile force in the carrier rope using a rope sensor, see Figure 1, is a commonly used method, however, it has certain limitations and drawbacks.

Under certain circumstances, the basic limitation is the way of obtaining tensile force values in the individual ropes of rope suspensions that use a higher number of ropes. As a result of the lead of the individual ropes into the grooves in the friction disc, it is required that the longitudinal axes of the carrier ropes are lead into the axes of the grooves of the friction disc at a maximum allowed, relatively small angle of attack. The limited size of the angle of attack of the rope into the groove in the friction disc is strictly secured by the choice of the pitch, i.e. the distance between the longitudinal axes of the adjoining ropes. Due to the low values of pitches of the longitudinal axes of the carrier ropes (in multi- rope systems), it is not possible to simultaneously mount the rope sensors on all cross- sections of the ropes in a limited space of the elevator shaft.
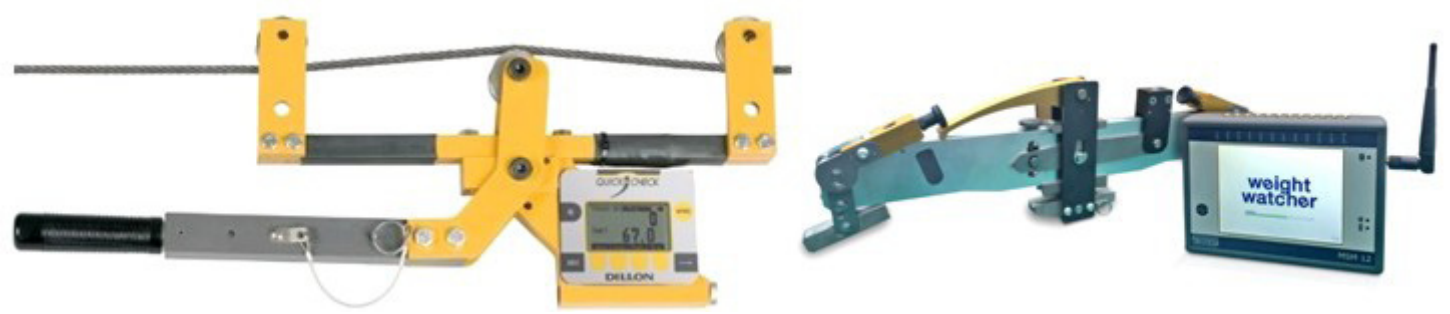

Figure 1. Rope sensor enables recording of tensile force in the rope [6] 


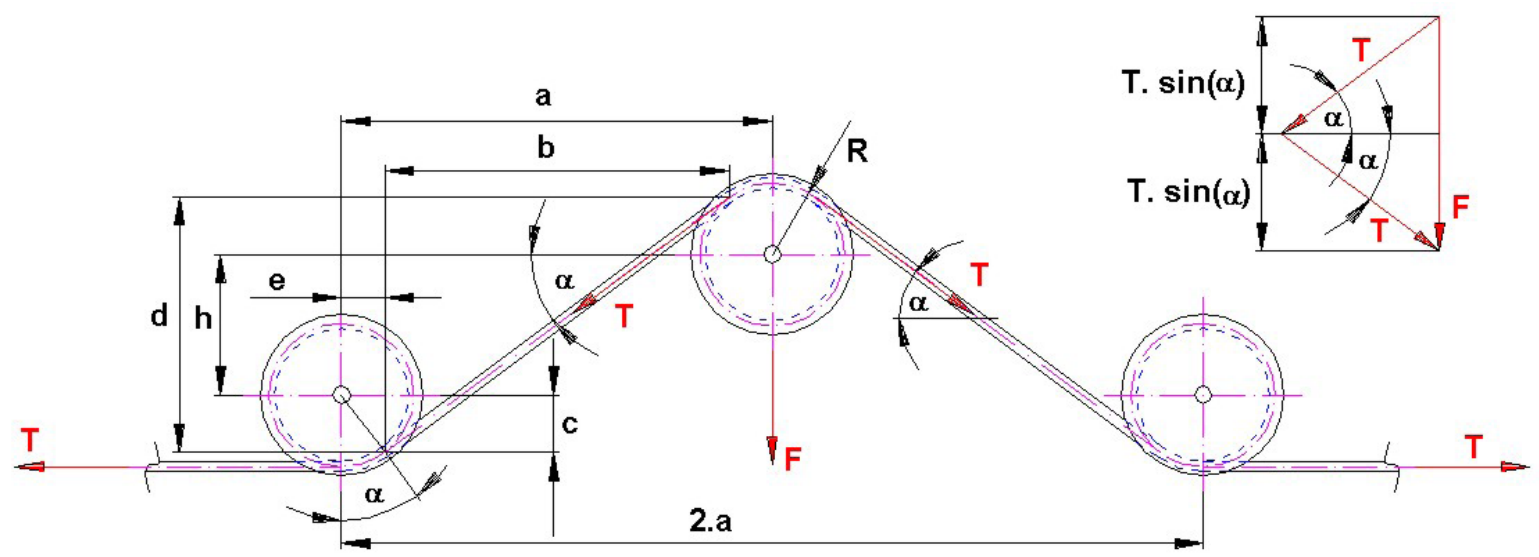

Figure 2. Principle of determining the tensile force with a rope sensor

Certain limitation in the use of the method for determining the tensile force in the carrier rope with a rope sensor can also be seen in the necessity to have this device mechanically attached on the ropes during the offset of the tensile forces. In a phase of operation, when new ropes are being installed in the elevator system and it is necessary to move the cage within the elevator shaft in order to offset tensile forces in the individual ropes, there is a risk of collision of the sensors attached to the ropes with the friction disc or other elements in the elevator shaft.

Another significant limitation presents the possibility of damaging the power cables to the tensometric sensors of the rope sensors, when there is a two-way movement of the elevator cage. When the cage is moving within the elevator shaft, then the carrier ropes and thus also the rope sensors, which are mechanically attached to the cross- sections of the ropes, are in motion as well.
The actual value of the tensile force acting on the rope axis is not directly measured by the rope sensor, it is determined proportionally from the normal force exerted, i.e. the force perpendicular to the rope axis. This normal force is a resultant of the components of the acting tensile forces in inclined sections of the measured rope, see Figure 2, and acts on the central contact member of the rope sensor. The actual value of the tensile force in the rope must therefore be determined by the comparative method. The accuracy of determining the actual tensile force in the rope is affected by the reshaping (deformation) of the rope sensor body and depends on the angle of inclination of the rope section and the distance of the gripping points of all contact points.

The above mentioned limitations and drawbacks can be eliminated using the device for offsetting tensile forces in elevator ropes described below.
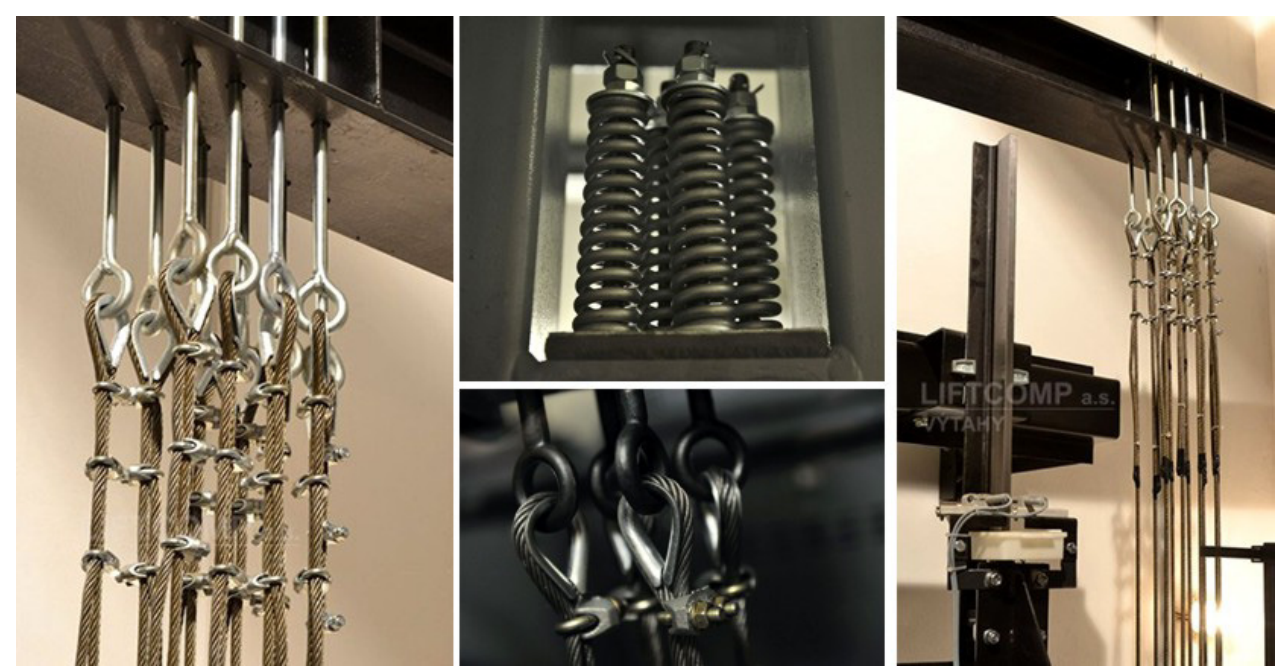

Figure 3. Suspension screws with openings to which the ends of the ropes are attached 

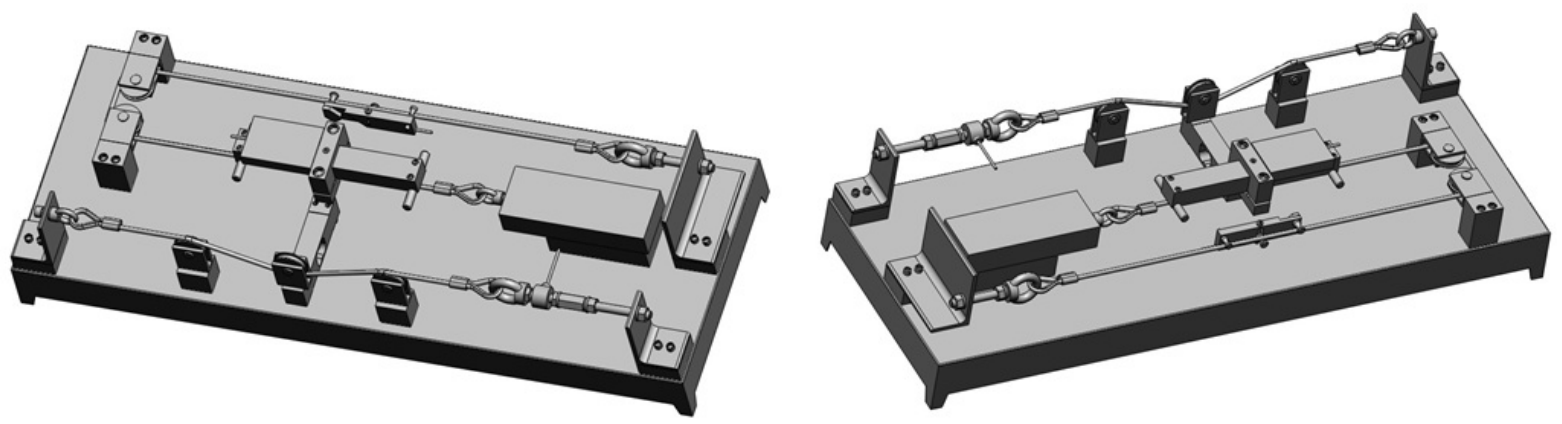

Figure 4. Design of prototype a device for offsetting tensile forces with rope sensor

\section{DEVICE FOR MEASURING THE TENSILE FORCE IN A ROPE}

A specific design and technical solution of the device for detecting and offsetting tensile forces in the elevator carrier ropes is illustrated in Figure 5. The described device is capable of continuously recording the time course of the instantaneous tensile forces, acting on elevator carrier ropes, when one free ends of the carrier ropes are mechanically attached to the openings of the suspension screws, which are mechanically tied to the bearing bracket, see Figure 3 .

Suspension screw 1, see Figure 6, is threaded through the opening in the bearing bracket 2 . On the thread of the suspension screw, above the up- per level of the bearing bracket, a bowl is pushed 3 in a defined direction, into which one end of the compression coil spring is inserted $\mathbf{4}$. The bowl $\mathbf{5}$ is placed upon the other end of the cylindrical coil spring 4 . The end section of the suspension screw $\mathbf{1}$ is attached with a washer $\mathbf{6}$ and the hexagonal nut $\mathbf{7}$ is screwed. This is how the suspension screw is locked against sliding out downwardly in the direction of the elevator shaft (due to the tensile force in the carrier rope) through the opening in the bearing bracket 2 .

The described device is a screw spindle $\mathbf{8}$, consisting of a double screw $\mathbf{9}$, single screw $\mathbf{1 0}$ and a cylindrical nut $\mathbf{1 1}$.

The double screw 9 (see Figure 6) is fitted with an outer trapezoidal isosceles single thread

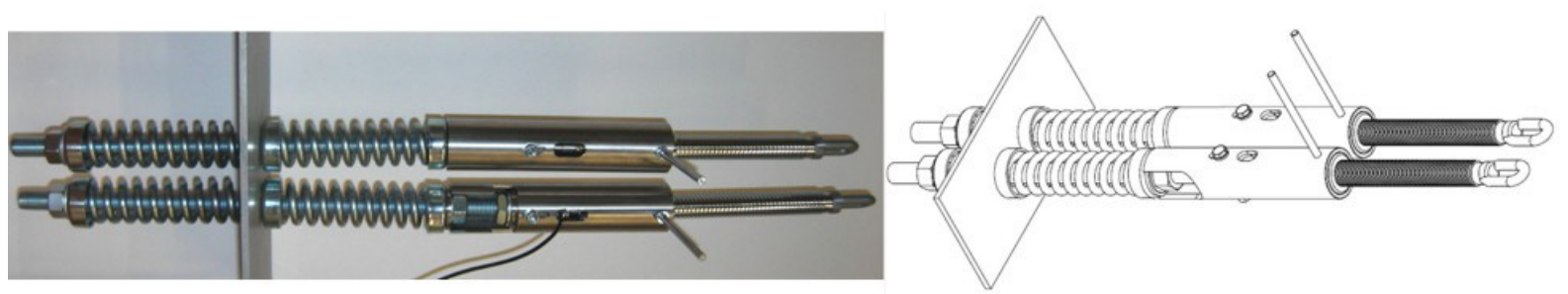

Figure 5. Realized prototype of a device for offsetting tensile forces in elevator ropes

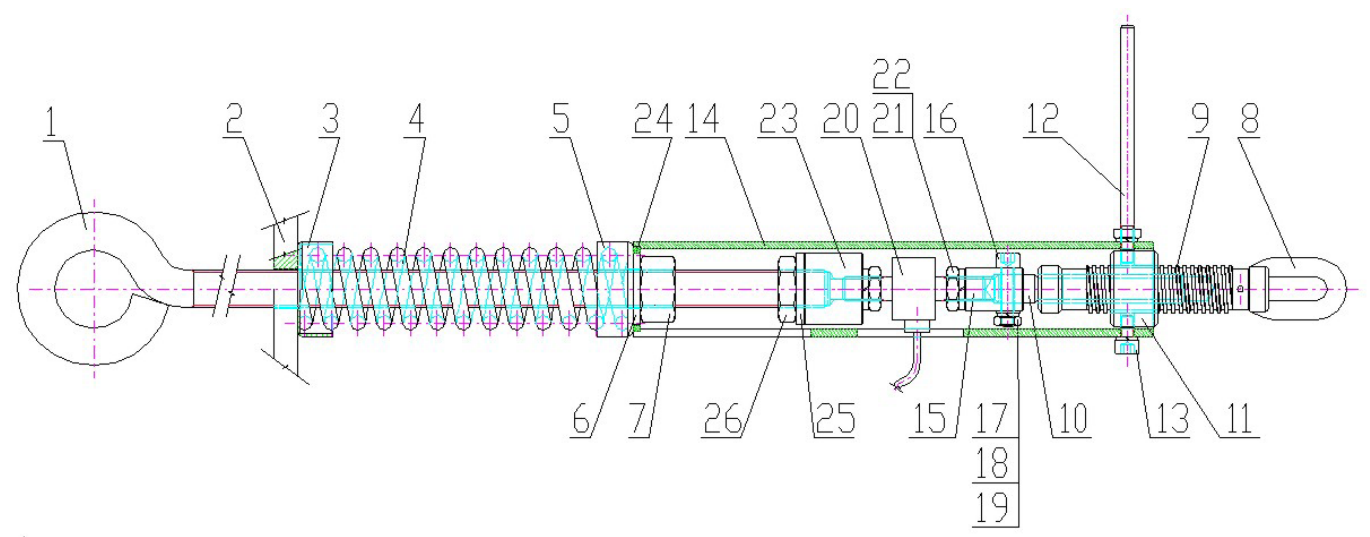

Figure 6. Construction design of a device for offsetting tensile forces in elevator ropes 
and inner trapezoidal isosceles single thread. The single screw $\mathbf{1 0}$ is fitted with trapezoidal isosceles single thread on its outer cylindrical surface. The cylindrical nut's outer surface $\mathbf{1 1}$ is fitted with two openings, into which the pins formed in the end sections, the cylindrical rods $\mathbf{1 2}$ and screws with cylindrical head and inner hexagon are inserted 13. Pins at the ends of the cylindrical rod $\mathbf{1 2}$ and the screw 13 in this way mechanically interconnect the cylindrical nut $\mathbf{1 1}$ with the body of the movable mechanical tension off-setter 14.

The single screw $\mathbf{1 0}$ is terminated by the cylindrical section with the created opening. Cylindrical part of the single screw $\mathbf{1 0}$ is inserted into the flange opening 15; preventing the rotation of the flange $\mathbf{1 5}$ in relation to the single screw $\mathbf{1 0}$ is provided by a screw $\mathbf{1 6}$, washer $\mathbf{1 7}$, adaptable washer 18 and a low hexagonal nut 19. A force sensor $\mathbf{2 0}$ is screwed into the threaded opening on the opposite side of the flange $\mathbf{1 5}$. The force sensor $\mathbf{2 0}$ is secured against loosening with an adaptable washer $\mathbf{2 1}$ and a low hexagonal nut $\mathbf{2 2}$.

The outer thread of the suspension screw 1 is screwed onto the inner thread of the hub 23; it is secured against loosening with a washer $\mathbf{2 5}$ and a low hexagonal nut 26. A nut $\mathbf{7}$ is screwed on the thread of the suspension screw 1 at the appropriate distance; see Figure 6, which leans against the bowl 5 over the washer $\mathbf{6}$. The outer diameter of the washer $\mathbf{6}$ is centered, in relation to the outer diameter of the body of the movable mechanical tension off-setter 14, by a centering ring 24 .

\section{DESCRIPTION OF OPERATION OF THE MOVABLE MECHANICAL TENSION OFF-SETTER}

In actual practice, the required number of suspension screws is threaded through the created openings in the bearing bracket, which are spaced apart in the desired, as small as possible pitch. One free end of the carrier rope is fastened with rope clamps over the rope eyepiece into the opening (created in the end section of the suspension screw, see Figure 3).

Tensile force acting on the suspension screw axis 1 (tensile force is exerted in practice by the relative loading, defined by the number of the carrier ropes used and the method of their use, in the respective branch of the carrier rope from the weight of the cage or the counterweight) is in the described device exerted by the same size onto the axis of the suspension screw 1 through the pressure of the coil spring in compressed state. Compression coil spring is compressed to the required force (required tensile force acting in the suspension screw axle) with a hexagonal nut. At the moment of achieving the desired axial force in one threaded rod $\mathbf{1}$, tensile force of a different size in the second suspension screw axle is exerted in a similar way.

Different values of tensile forces exerted by different forces in the carrier ropes, see Figure 7, and thus by pre-tensioning of the cylindrical compression coil springs $\mathbf{4}$ are gauged by the tensile

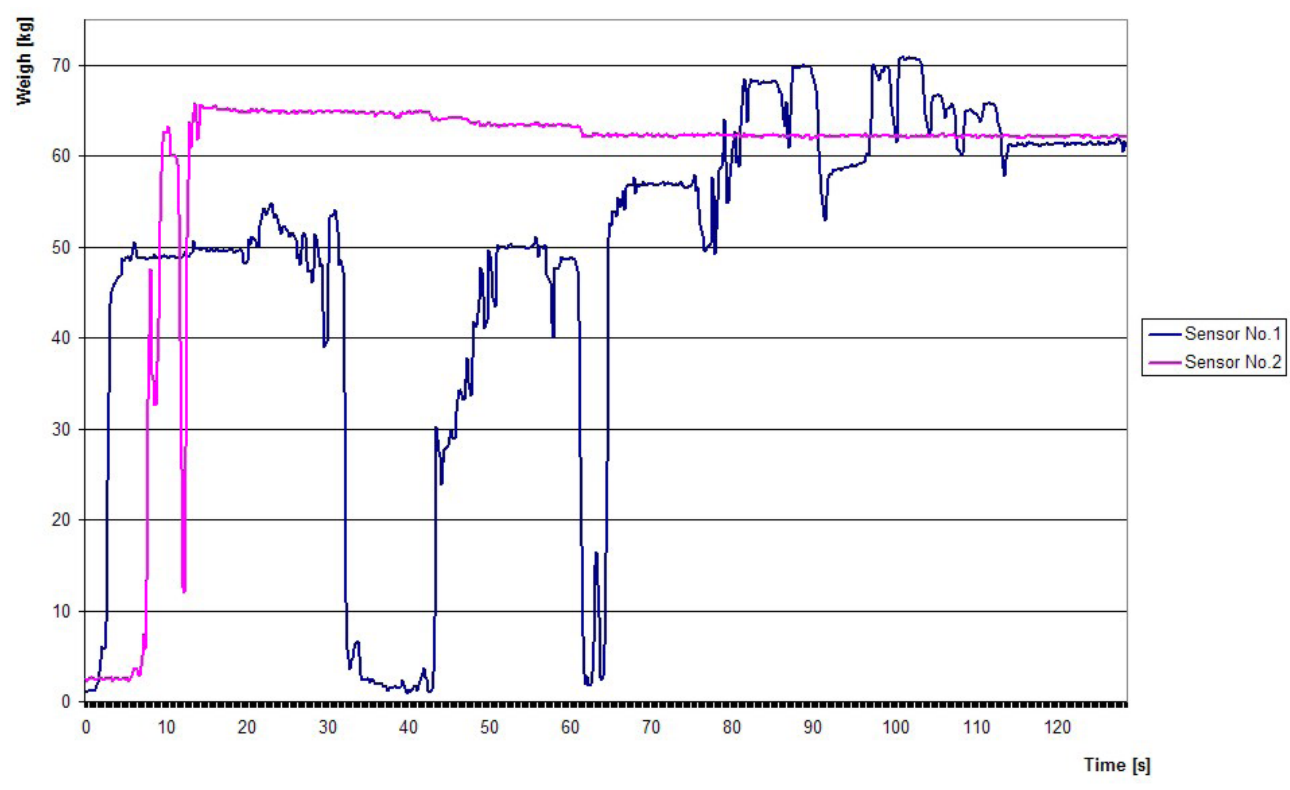

Figure 7. The process of the two tensile forces measured by the DEWEsoft programme in the tension equalizers of ropes and rope lifts 
force sensors 20, see Figure 6. In order to achieve the same value of the tensional force in the carrier ropes, the double screw 9 of the individual screw spindle $\mathbf{8}$ is rotated, which leads to a compression (release) of the given compression coil spring 4 . Compression or release (given by the purpose of rotation of the suspension screw 9 of the individual screw spindle 8) of the individual compression coil spring $\mathbf{4}$ are due to the design of the movable mechanical tension off-setter, namely the fixed length distance (at a given moment) between the plane of placement of the cylindrical nut $\mathbf{1 1}$ (the link is provided by the pins inserted into the openings created in the end sections of the cylindrical rod 12 and screws with cylindrical head and inner hexagon 13) and the upper surface of the bearing bracket $\mathbf{2}$.

In the lower part of the body $\mathbf{1 4}$ of the movable mechanical tension off-setter, there is a mounting (neck- down), into which a centering ring is inserted 24, see Figure 5. The inner diameter of the centering ring $\mathbf{2 4}$ thus defines a play between the outer dimensions of the washer $\mathbf{6}$ and the inner diameter of the body mounting $\mathbf{1 4}$. At the moment of rotation (in a given direction) of the double screw $\mathbf{9}$ of the individual screw spindle $\mathbf{8}$, there is an extension (shortening) of the distance between the plane of placement of the cylindrical nut $\mathbf{1 1}$ (in relation to the body 14) and the upper surface of the bearing bracket 2 . The mutual mechanical connection of the hub $\mathbf{2 3}$ with the suspension screw $\mathbf{1}$ and the screw spindle $\mathbf{8}$, which is through the cylindrical nut $\mathbf{1 1}$ mechanically interconnected with the body $\mathbf{1 4}$ of the movable mechanical tension off-setter, gains one degree of freedom. That means the hub $\mathbf{2 3}$ allows a shift in relation to the longitudinal axis of the body $\mathbf{1 4}$.

Based on the assumptions described above, at the moment of rotation (in a given direction) of the double screw 9 of the individual screw spindle $\mathbf{8}$, there is an extension (shortening) of the distance between the plane of placement of the cylindrical nut $\mathbf{1 1}$ and the centering ring $\mathbf{2 4}$, which is inserted into the mounting in the lower part of the body 14 . The centering ring 24 leans against the outer surface of the bowl $\mathbf{5}$. At the moment of rotation of the double screw $\mathbf{9}$ of the screw spindle $\mathbf{8}$ in a given direction, the bowl $\mathbf{5}$ is being pushed away by the centering ring $\mathbf{2 4}$ (the distance between the cylindrical nut $\mathbf{1 1}$ and the surface of the bowl $\mathbf{5}$ is extending), thereby causing compression of the coil spring 4 (when rotating the double screw 9 of the screw spindle $\mathbf{8}$ in the reverse direction, the bowl $\mathbf{5}$ is being pushed to the centering ring $\mathbf{2 4}$ (the distance between the pins of the cylindrical nut $\mathbf{1 1}$ and the surface of the bowl $\mathbf{5}$ is shortening), thereby causing release of the coil spring 4 ).

Above the bowl 5, the washer is threaded $\mathbf{6}$ on the suspension screw $\mathbf{1}$ followed the hexagonal nut 7. By tightening (loosening) the nut 7 , it is possible to fix the compressed (released) spring 4 in the desired state. Since the value of the tensional force in the individual suspension screw 1 can be continuously gauged and compared to the value of the tensional force in the adjoining suspension screw $\mathbf{1}$ using tensional force sensors $\mathbf{2 0}$ (in practice in adjoining suspension screws), the above mentioned procedure enables achieving the same values (offsetting the tensional forces in the individual carrier ropes) of the tensional forces exerted in the suspension screws from the initially differently set values caused by the different pre-tensioning of the cylindrical compression coil springs 4 .

\section{CONCLUSIONS}

The content of the contribution is a theoretical description of the operation of the device.

Detailed information on the measuring device that reads the signals of measuring sensors and the environment that graphically records the time course of the measured signals is provided in contribution titled ,Apparatus producing an even distribution of strain into carriers", see $[4,6,5]$.

The possibility to measure the real value of the tensile force in the partial cross section of the carrying lift rope, to compare this to the value of the tensile force of the adjacent carrying rope and consequently, on the basis of the knowledge of difference of both (or more) values of tensile forces, to equalize both values in mechanical way (by turning the nut attached to the rope hinge or to the rod), it enables to use the described apparatus in practice to set the appropriate rope tension in multi-roped hinges.

\section{REFERENCES}

1. Vomočil M. ČSN EN 81-1+A1 (274003) Safety rules for the construction and installation of lifts - Part 1: Electric lifts. MOVA Karlovy Vary, 1999. In Czech.

2. ČSN EN 81-20 Safety rules for the construction 
and installation of lifts - Lifts for the transport of persons and goods - Part 20: Passenger and goods passenger lifts. Úřad pro technickou normalizaci, metrologii a státní zkušebnictví, Praha, 2015 - In Czech.

3. Dvořák J. ČSN EN 81-50 Safety rules for the construction and installation of lifts - Examinations and tests - Part 50: Design rules, calculations, examinations and tests of lift components. CTN Unie výtahového průmyslu ČR. Úřad pro technickou normalizaci, metrologii a státní zkušebnictví: Ing. Jaroslav Zajíček, Praha, 2015 - In Czech.

4. Hrabovský L. Apparatus producing an even distribution of strain into carriers. WM CAUS 2017. IOP Conference Series-Materials Science and Engineering. Volume: 245, Article Number: UNSP 072047, DOI: 10.1088/1757-899X/245/7/072047.

5. 5. Hrabovský L., Michalik P. A tension equalizer in lift carrying ropes. Advances in Science and Technology Research Journal 4, 2017, 236-332.

6. http://www.tensionmeters.com/design.htm

7. Molnar V., Boroska J., Decmanova J. Mechanical properties of steel rope wires - quality test assurance. Acta Montanistica Slovaca 15, 2010, 23-30.

8. Kmet S., Stanova E., Fedorko G., Fabian M., Brodniansky J. Experimental investigation and finite element analysis of a four-layered spiral strand bent over a curved support. Engineering Structures 57, 2013, 475-483.

9. Stanova E., Fedorko G., Kmet S., Molnar V., Fabian M. Finite element analysis of spiral strands with different shapes subjected to axial loads. Advances in Engineering Software 83, 2015, 45-58.

10. Fedorko G., Stanova E., Molnar V., Husakova N., Kmet S. Computer modelling and finite element analysis of spiral triangular strands. Advances in Engineering Software 73, 2014, 11-21.

11. Ivanco V., Kmet S., Fedorko G. Finite element simulation of creep of spiral strands. Engineering Structures 117, 2016, 220-238.

12. Debski H., Teter A., Kubiak T., Samborski S. Local buckling, post-buckling and collapse of thin-walled channel section composite columns subjected to quasi-static compression. Composite Structures, 136, 2016, 593-601.

13. Palencar R., Duris S., Ranostaj J. Conclusions and some comments on the calculation of uncertainty when constructing a temperature scale. Measurement Techniques, 54(8), 2011, 910-920.

14. Garbacz T., Jachowicz T., Gajdoš I., Kijewski G. Research on the influence of blowing agent on selected properties of extruded cellular products. Advances in Science and Technology Research Journal, 9(28), 2015, 81-88. 